\title{
Les sans-papiers, le chef et l'ethnographe
}

Perceptions de genre, de classe et de race sur un petit chantier de Barcelone

The undocumented workers, their boss and the ethnographer. The perceptions of gender, class and race in a small Barcelona building site

\section{Yann Beldame}

\section{(2) OpenEdition}

Journals

Édition électronique

URL : http://journals.openedition.org/conflits/18851

DOI : 10.4000/conflits. 18851

ISSN : $1777-5345$

Éditeur :

CCLS - Centre d'études sur les conflits lilberté et sécurité, L'Harmattan

Édition imprimée

Date de publication : 8 juillet 2014

Pagination : 65-86

ISBN : 978-2-343-04155-1

ISSN : 1157-996X

Référence électronique

Yann Beldame, «Les sans-papiers, le chef et l'ethnographe », Cultures \& Conflits [En ligne], 93 |

printemps 2014, mis en ligne le 02 juillet 2015, consulté le 30 mars 2021. URL : http://

journals.openedition.org/conflits/18851 ; DOI : https://doi.org/10.4000/conflits.18851 


\title{
Les sans-papiers, le chef et l'ethnographe
}

\author{
Perceptions de genre, de classe et de race sur un petit chantier \\ de Barcelone
}

\section{Yann BELDAME}

Yann Beldame est Docteur en anthropologie sociale et ethnologie (EHESS) et ATER à l'Université Montpellier 1. Sa thèse, achevée en 2010, s'intitule "Sans papier, sans destin. Essai d'ethnographie critique dans l'Espagne de l'immigration" (sous la direction d'Alban Bensa).

et article s'appuie sur une enquête de terrain qui visait à retracer la vie de
quelques migrants « sans-papiers » récemment arrivés en Espagne et tra-
vaillant dans le secteur du bâtiment. L'enquête s'est déroulée à Barcelone et a
été réalisée entre le mois de février 2004 et le mois d'avril 2005. Quatre per-
sonnages principaux ont participé aux situations décrites ici : Manuel, Luis,
Raoul et bien sûr l'anthropologue lui-même. Je me suis attaché à décrire
quelques-unes de nos interactions en insistant tout particulièrement sur les
catégories qui furent mobilisées pour créer entre nous de la différence. Mais
avant d'en venir à l'analyse de ces logiques classificatoires, je commencerai par
une brève présentation des acteurs et des circonstances dans lesquelles je les ai
rencontrés ${ }^{1}$.

Après avoir obtenu un DEA ${ }^{2}$ en septembre 2003, je choisis de partir pour 4 mois à Barcelone en tant qu'étudiant Erasmus. Je me dis que ce serait une bonne occasion de parfaire mon castillan et surtout de me donner encore un peu de temps avant de commencer une thèse, pour laquelle je n'avais pas encore arrêté le thème de façon définitive. Lorsque j'arrivai à Barcelone le 11 février 2004, je n'avais toujours pas l'intention de réaliser une enquête ethnographique sur les maçons sans-papiers. Ma situation financière ne me laissait d'autre choix que celui d'opter pour une chambre en sous-location à un prix modique. Manuel, alors âgé de 21 ans, était l'un des sous-locataires qui partageait avec moi l'appartement. Il avait quitté le Paraguay depuis deux mois. Il avait fait le

1. Dans un souci de mise en forme, $j$ 'ai choisi de restituer en italique les extraits de mon journal de terrain.

2. Il s'agit du DEA «Sciences sociales » qui était rattaché à la fois à l'EHESS et à l'ENS. 
voyage avec sa cousine Nuri qu'il ne rencontrait que le week-end car, le reste de la semaine, elle cuvrait en tant qu' "interna " (employée de maison) chez un entrepreneur du secteur postal. Au moment où je connus Manuel, il avait encore des rêves. Il avait voyagé en Espagne pour gagner de l'argent et en épargner une partie conséquente. Il ambitionnait de construire une "grande maison "dès son retour au Paraguay et quand on lui demandait à quel moment il soubaitait retrouver son pays, il répondait invariablement "d'ici trois ou quatre ans". Il affirmait que cette petite fortune lui servirait à poursuivre les études de gestion qu'il avait entamées deux ans plus tôt à l'université d'Asunción. Une fois qu'il aurait obtenu son diplôme, il pensait aussi ouvrir un petit commerce ("n'importe quoi, pourvu que ça rapporte»). Le 29 décembre 2003, lorsque Manuel et sa cousine Nuri atterrirent à Barcelone, ils ne pouvaient compter que sur eux-mêmes. Il n'y avait pas d'amis paraguayens qui puissent les accueillir ni de réseau familial sur lequel s'appuyer. Pourtant, quatre jours après leur arrivée, Manuel était déjà embauché en tant que maçon pour rébabiliter des appartements. Il se baladait avec Nuri "juste là, aux abords des Ramblas ", lorsque Nuri, plutôt volubile, aborda un bomme à l'apparence sud-américaine pour lui demander où ils pourraient trouver du travail. Ce dernier lui parla avec une apparente bonbomie en lui expliquant qu'il avait émigré du Pérou vingt ans plus tôt, au même âge que Manuel. Au bout de quelques instants, il proposa à Manuel de l'embaucher sur des chantiers. Les procédés d'embauche que Manuel venait d'expérimenter lui paraissaient certes non conventionnels, mais il n'avait d'autre choix que celui d'accepter. Il avait emprunté quelques milliers d'euros pour payer son voyage et pour assurer sa pitance durant les premiers jours de son périple. Cet emploi lui permettrait de mettre à jour sa situation financière. Il n'avait jamais tenu une truelle de sa vie mais il n'avait pas vraiment le temps ni le loisir de s'en inquiéter. Quelques jours plus tard, le voilà précipité au milieu des pots de peinture, des anges et des sacs de ciment.

L'appartement qu'il allait rébabiliter était situé rue Nou de la Rambla, dans le quartier populaire de Parallel. Dans cette partie de la ville, si le bâti avait gardé son côté ouvrier hérité de l'industrialisation du XIXème siècle, les usines en revanche n'existaient plus et le quartier accueillait à présent un nombre croissant d'immigrés. Les bars équatoriens et les épiceries pakistanaises foisonnaient. Ces petits commerces remplaçaient peu à peu les salles pornographiques, bars, théâtres, cabarets et autres cafés-concerts qui avaient constitué, au début du XXème siècle, la splendeur de ce Montmartre barcelonais. Dans la seule avenue oì travaillait Manuel, il y avait quatre autres chantiers d'ampleur semblable. Très vite, je me mis à lui rendre visite ${ }^{3}$ et lui prêtai main forte

3. Dans les premiers temps, je ne passais pas plus d'une heure sur les chantiers, toujours au moment où le chef n'y était pas. Devant les autres maçons, Manuel m'avait présenté comme étant un «ami Français ». Lorsque je leur annonçai que je souhaitais travailler à leur côté, tous me le déconseillèrent fermement, prétextant que le chef payait ses ouvriers en retard et que ce type d'emploi n'était pas fait pour moi. 
pour repeindre des pans de mur. Au bout de quelques semaines, je réussis à convaincre le chef de m'embaucher en tant que manouvre. Je ne cachai pas mon ambition intellectuelle à mes collègues de travail et au chef. Je leur dis que je soubaitais faire de cette expérience "un livre sociologique sur les immigrés".

Dès les premiers jours, je travaillai auprès de Luis, un Péruvien de 53 ans qui ne totalisait que quelques semaines de présence à Barcelone. Il affirmait s'être déplacé en Espagne "pour y apprendre les techniques de transformation des aliments ". Dès son retour au Pérou, il soubaitait développer une entreprise de confitures et de jus de fruits qu'il avait récemment créée à Lima. Durant la plus grande partie de sa vie, Luis avait exercé le métier de barman au sein d'hôtels cinq étoiles et c'est en profitant d'un championnat international de cocktails qui se déroulait à Séville qu'il avait pu "passer " la frontière. À la différence de Manuel, Luis avait quelque peu préparé sa venue à Barcelone. Au Pérou, il avait déjà rencontré Raoul, le propriétaire de l'entreprise pour qui nous travaillions. Le père de Raoul était l'un de ses amis. Raoul lui avait promis de l'embancher dès son arrivée en Espagne. Il l'avait même aidé à trouver un logement. Au cours des premiers mois, Luis vivait en effet chez une cousine de Raoul qui lui sous-lonait une chambre.

Raoul avait 37 ans et vivait en Espagne depuis 19 ans. Il avait quitté le Pérou après une brève carrière dans l'armée. Le Sentier Lumineux menaçait alors les militaires et son père avait préféré l'envoyer en Espagne pour le "mettre à l'abri ». Dès son arrivée, Raoul travailla sur les chantiers barcelonais où, au fil des années, il avait réussit à améliorer ses compétences de maçon et à noner de nombreuses relations professionnelles. Au bout de sept ans, il décida de créer sa propre entreprise qu'il spécialisa dans la rébabilitation d'appartements, un domaine pour le moins lucratif depuis que la municipalité barcelonaise soutenait les propriétaires désireux d'entretenir leurs biens immobiliers. À présent, Raoul avait acquis la nationalité espagnole et maîtrisait le catalan qu'il prétendait parler " avec les architectes".

Enfin, si dans cet article j'ai soubaité décrire les diverses formes de différenciation à l'œuvre sur les chantiers, ça n'est pas sans oublier qu'elles se déployaient en présence d'un jeune anthropologue français de 26 ans, perçu par ses hôtes en tant que «blanc» et dont le projet de recherche était connu de tous.

L'immersion ethnographique n'est pas simplement prise ici comme un outil méthodologique permettant d'accéder aux catégories indigènes de classement de soi et des autres; elle est considérée aussi comme un objet d'étude à part entière. Car, sur les chantiers, la présence inhabituelle d'un jeune anthropologue français suscita de nombreuses réactions de la part des maçons sanspapiers qui ont elles-mêmes fait réagir le chercheur. «Transfert » et « contretransfert » dirait Georges Devereux ${ }^{4}$, pour qui les « perturbations » dues à l'existence de l'observateur, lorsqu'elles sont correctement exploitées, sont les 
pierres angulaires d'une science du comportement authentiquement scientifique » 5. Par ailleurs, si j'ai considéré que l'étude du dispositif et des relations d'enquête faisait partie de l'enquête, ce n'est pas pour mettre au centre du récit le personnage héroïque et subséquemment narcissique du chercheur mais plutôt pour prévenir le discours "allochronique » identifié par Johannes Fabian dans Le temps et les autres ${ }^{6}$. Mes interlocuteurs et moi partagions en effet le même temps ; or la reconnaissance de cette "co-temporalité » constitue l'une des conditions de possibilité de toute forme d'anthropologie.

\section{Orientation méthodologique}

Ayant été activement engagé au sein des chantiers, je peux considérer qu'il y eut une "prépondérance de la participation sur l'observation " 7 . Mais si la méthode que je mis en œuvre fut une forme de " participation observante », c'est avant tout parce que dans mon cas « I'accent passe de l'observation de l'autre à l'observation de la relation humaine entre soi, en tant qu'ethnographe, et l'autre ${ }^{8}{ }^{8}$. J'estime à ce titre que les situations communicationnelles survenues au cours de l'enquête entre l'ethnographe et ses hôtes furent source et moteur de savoir ${ }^{9}$. Pour autant, peu de conversations purent être enregistrées. Ayant décidé initialement d'utiliser le magnétophone, je le laissai pourtant ensuite au fond d'un tiroir la majeure partie du temps. Je le considérai comme le signe de mon extériorité sociale et sans doute éprouvai-je un certain malaise à rendre visibles les véritables raisons de ma présence parmi eux. Ce détail technique eut pourtant son effet au moment de l'écriture, puisque vous remarquerez au fil du texte que les dialogues prennent peu de place et qu'ils furent la plupart consignés sans être enregistrés. Hormis pour certains faits biographiques concernant Luis et Raoul et produits lors d'entretiens enregistrés, j'ai utilisé dans le reste du récit l'ensemble des écrits élaborés par mes soins jour après jour. Après avoir passé une journée sur les chantiers, ou sitôt que je venais de rencontrer Luis et Manuel, je me plongeais immédiatement dans l'écriture détaillée de ce qui venait d'arriver et d'être dit, et cela jusqu'aux derniers instants passés à leurs côtés. Mon journal de terrain ressemble donc à un amas de souvenirs, où la mémoire fut mobilisée aussitôt le surgissement des événements et sans qu'une nuit ou qu'un sommeil n'ait pu les séparer. Ma maîtrise du castillan étant très imparfaite, je dus l'apprendre comme n'importe quel Français, c'est-à-dire au prix d'un effort conséquent. Durant les premières semaines d'enquête, je n'étais toujours pas bilingue. Or, même si la maîtrise imparfaite du castillan put constituer un biais dans la production des données, elle put également en être un moteur puisqu'elle fut maintes fois l'occasion de demander à mes hôtes de préciser certaines de leurs déclarations et de m’assurer par là du sens qu'ils leur attribuaient.

4. Devereux G., De l'angoisse à la méthode dans les sciences du comportement, Paris, Flammarion, 2012 (1980 pour la 1ère édition française ; 1967, pour la 1ère édition anglaise).

5. Ibid, p. 30.

6. Fabian J., Le temps et les autres, Comment l'anthropologie construit son objet, Toulouse, Anacharsis, 2006.

7. Soulé B., «Observation participante ou participation observante ? Usages et justifications de la notion de participation observante en sciences sociales ", Recherches qualitatives, 27-1, 2007, pp. 127-140.

8. Ibid, p. 131 .

9. À ce sujet, on peut lire notamment Emerson R., «Le travail de terrain comme activité d'observation. Perspectives ethnométhodologistes et interactionnistes », in Cefaï D. (ed.), L'enquête de terrain, Paris, La Découverte/MAUSS, 2003. À la page 410, Emerson écrit : «Le travail de terrain est nécessairement de nature interactionnelle et la présence de l'enquêteur a des conséquences dans la vie des enquêtés. Les solutions à la réactivité ne sont pas dans la régulation, la restriction ou la suppression des interactions sur le terrain. Elles réclament que l'on devienne sensible et réceptif à la façon dont les protagonistes se perçoivent et se traitent les uns les autres. Le chercheur est "source de résultats, non pas de contamination de ceuxci” ». 


\section{L'ethnographe à l'intersection des rapports de pouvoir}

Quelques mois après ma participation au chantier, lorsque je relisais mes notes de terrain, je me rendis compte que nos relations s'étaient toutes structurées autour de perceptions de «genre », de « classe » et de « race ». Ce n'est pourtant pas à l'une de ces catégories que je souhaitais m'intéresser particulièrement, mais plutôt à leur intersection et à leur imbrication. Par exemple, la mise en scène d'une forme de vaillance sur les chantiers ne relevait pas seulement de la constitution d'une identité d' «immigré »; elle participait aussi d'un codage sexuel des rapports de classe. Il s'agissait donc d'explorer comment s'étaient construits et articulés, au sein du chantier, des rapports de genre, de classe et de race et comment ces rapports avaient produit une configuration spécifique du pouvoir qu'on ne saurait représenter sur une échelle unidimensionnelle. Pour ce faire, j'utilise ici le concept d' 'intersectionnalité », formulé pour la première fois en 1991 par la juriste Kimberlé Crenshaw ${ }^{10}$. Ce concept est ici appliqué dans le cadre d'une production de données ethnographiques. Je le rends opératoire en anthropologie en l'intégrant dans une approche goffmanienne de l'interaction sociale. En m'appuyant en effet sur les situations d'« embarras », finement analysées par Erving Goffman dans Les rites d'interaction ${ }^{11}$, je centre mon attention sur les moments de trouble ${ }^{12}$ que provoquait la présence d'un anthropologue «blanc» dans un univers ouvrier racialisé.

Avec l'idée d'intersectionnalité, il ne s'agit pas de concevoir de façon arithmétique les rapports sociaux de sexe, de classe et de race pour montrer comment la situation des sans-papiers résulte d'une double ou d'une triple oppression. Au lieu d'additionner simplement les propriétés sociales stigmatisées des individus, je souhaite déplacer mon regard vers les situations d'interaction où les acteurs utilisent et font jouer ces propriétés, notamment dans des logiques de compensations parfois inattendues ${ }^{13}$. À ce titre, il est utile de rappeler, comme le fait Michel Foucault ${ }^{14}$, que les rapports de pouvoir ne sont jamais simplement répressifs. Dans La volonté de savoir ${ }^{15}$, il écrit en effet que

10. Crenshaw K., "Mapping the margins: Intersectionality, identity politics and violence against women", Stanford Law Review, 43, 1991, pp. 1241-1298. Cet article fut traduit en Français dans les Cahiers $d u$ genre : Crenshaw K. et Bonis O., "Cartographies des marges : intersectionnalité, politique de l'identité et violences contre les femmes de couleur ", Cabiers $d u$ Genre, 39-2, 2005, pp. 51-82.

11. Goffman E., «L'embarras et l'organisation sociale » in Les rites d'interaction, Paris, Éd. de Minuit, 1973, pp. 87-100.

12. Sur l'utilité des situations de trouble en anthropologie on peut lire : Chauvier É., «Préface. Le livre du voleur. De l'importance du trouble en anthropologie ", in Congoste M., Le vol et la morale. L'ordinaire d'un voleur, Toulouse, Anacharsis, 2012. À la page 7, Éric Chauvier écrit qu'il existe une « heuristique du trouble » car les «situations de troubles expriment la quête essentielle de l'anthropologue, à la fois sa démarche et sa finalité, ici confondues à une suite d'anomalies révélant en creux les règles d'une vie sociale».

13. Jaunait A. et Chauvin S., " "Représenter l'intersection". Les théories de l'intersectionnalité à l'épreuve des sciences sociales », Revue Française de science politique, 62-1, 2012, pp. 5-20.

14. Foucault M., « Le sujet et le pouvoir », in Dits et écrits II, 1976-1988, Paris, Gallimard, 2001.

15. Foucault M., Histoire de la sexualité. I : La volonté de savoir, Paris, Gallimard, 1976. 
« les relations de pouvoir ne sont pas en position de superstructure, avec un simple rôle de prohibition ou de reconduction ; elles ont, là où elles jouent, un rôle directement producteur »16. Il y a donc chez Michel Foucault une positivité du pouvoir car pour lui les sujets se constituent dans et par les relations de pouvoir. Dès lors, son concept de «subjectivation » me permit de qualifier la marge de manœuvre des maçons immigrés avec qui je vécus et travaillai durant mon enquête. Intermédiaire entre le subjectif et l'objectif, mais aussi entre l'individuel et l'institutionnel, l'idée de subjectivation démontre que Luis et Manuel pouvaient être déterminés tout en restant agissants. Car c'est au cœur même des rapports sociaux du chantier que ces immigrés recomposaient jour après jour un « rapport de soi à soi » 17 , constitué de mises à distance avec le chef et ses sommations mais aussi avec moi-même, ce Français à leurs yeux sans doute un peu bizarre qui venait d'accepter un emploi qui n'était pas fait pour lui.

\section{Illégalité, précariat et discrimination dans l'Espagne de l'immigration}

Les catégories que j'ai utilisées avec les enquêtés au cours de nos échanges n'étaient pas hors du temps mais s'inscrivaient dans un contexte politico-historique précis, celui de l'Espagne de l'immigration du début des années 2000. La signification de ces catégories n'est donc pas «transhistorique » comme le rappelle Joan Scott ${ }^{18}$. Il faut en effet «mettre au jour les prémisses sur lesquelles reposent les catégories qui organisent nos identités (personnelle, sociale, nationale), (...) leur donner une histoire, (...) les situer dans le temps et $(. .$.$) les soumettre à examen » 19$.

Au moment de l'enquête, l'Espagne était déjà devenue un important pays d'immigration. Entre 1997 et 2004, le nombre d'étrangers avait été multiplié par 4,8, passant de 637000 à près de 3,7 millions. Au cours de ces sept années, l'Espagne était parvenue au niveau des pays d'immigration ancienne, comme la France ou l'Allemagne 20. Cette évolution démographique n'a pas suscité de la part du gouvernement espagnol une ligne politique homogène ${ }^{21}$. Certes,

16. Ibid, p. 124.

17. Foucault M., L’herménentique du sujet, Cours au Collège de France 1981-1982, Paris, Gallimard/Seuil , 2001, pp. 241-242. Il écrit notamment : « il n'y a pas d'autre point, premier et ultime, de résistance au pouvoir politique que dans le rapport de soi à soi ».

18. Scott J., Théorie critique de l'histoire. Identités, expériences, politiques, Paris, Fayard, 2009, p. 48.

19. Ibid., p. 62.

20. L'immigration internationale vers Madrid et Barcelone, les deux principales aires métropolitaines espagnoles, s'est multipliée par dix. Si nous prenons le cas de Barcelone, la population étrangère est passée de 23720 personnes en 1991 à 202489 en janvier 2004 (Mairie de Barcelone, 2004), soit environ $15 \%$ de la population totale : une réalité socio-démographique proche de celle d'autres grandes villes européennes comme Paris, Bruxelles, Londres ou Berlin où le nombre d'étrangers oscille entre 15 et $30 \%$ de la population totale.

21. Ces contradictions dans la politique migratoire espagnole trouvèrent leur expression la plus évidente dans deux modifications du cadre légal de l'immigration qui se produisirent en 2000 : le passage à la loi organique 4/2000 d'abord puis la contre-réforme initiée par le Partido 
l'adhésion de l'Espagne à l'espace Schengen 22 le 25 juin 1991 a marqué son ralliement à une politique commune des visas. Cependant l'irrégularité de la situation juridique des migrants n'a pas toujours été prioritaire dans sa politique d'immigration. Celle-ci a généralement oscillé entre élaboration de textes législatifs d'ouverture vis-à-vis des immigrés, accompagnée de plusieurs vagues de régularisations, et instruments davantage restrictifs et sécuritaires. La contre-réforme amorcée par le Partido Popular en 2000, substituant la loi organique $8 / 2000$ à la loi organique 4/2000, a signifié le durcissement des conditions d'accueil des étrangers : la loi $8 / 2000$ revenait en effet à une conception instrumentale de l'immigration dans la mesure où l'immigré n'était reconnu juridiquement qu'en tant qu'étranger dont la présence est purement conjoncturelle, c'est-à-dire inscrite dans un laps de temps où le marché du travail en a besoin. Le changement politique survenu en mars 2004 n'a pas radicalement transformé cet état des lois ${ }^{23}$. Au-delà de la sixième vague de régularisation des sans-papiers survenue en 2005 et d'une méthode plus ouverte au dialogue avec les partenaires sociaux, les bases des anciennes orientations étaient sensiblement les mêmes : la loi d'extranéité de 2003 demeurait à l'identique et le nouveau règlement maintenait la « situation nationale de l'emploi » comme le critère essentiel permettant l'admission de nouveaux travailleurs immigrés.

Ces dispositifs d'accueil des étrangers pour le moins restrictifs et « inhospitaliers » 24 s'inscrivent dans une politique d'immigration qui se déploie à l'échelle européenne. Comme le rappelle en effet Andréa Réa : «le maintien des nouveaux venus dans un statut de séjour irrégulier ou précaire est devenu une composante de la politique européenne d'immigration » 25 . En outre, cette politique d'immigration répond à des politiques économiques néolibérales qui ont accompagné une transformation sociétale de plus grande ampleur : le passage d'une société du «salariat », caractérisée par le plein emploi, la prééminence des contrats à durée indéterminée, les garanties du droit du travail qui lui sont liées et les protections de l'État social, à une société du « précariat » 26,

Popular avec la loi organique 8/2000. À ce sujet lire: Gabrielli L., La construction de la politique d'immigration espagnole : ambiguïtés et ambivalences à travers le cas des migrations onest-africaines, Thèse pour le doctorat en Science politique, Sous la direction de Daniel C. Bach, Sciences Po Bordeaux, 2011.

22. L'adhésion espagnole à l'espace Schengen s'est faite, conjointement au Portugal, par la signature de l'accord, le 25 juin 1991. L'espace Schengen vise à instaurer un espace de libre circulation des personnes entre les États signataires et associés tout en garantissant une protection renforcée aux frontières extérieures de l'espace.

23. José Luis Rodríguez Zapatero (PSOE : Parti Socialiste Ouvrier Espagnol) fut élu à la tête du gouvernement espagnol suite aux élections générales du 14 mars 2004.

24. Fassin D., Morice A., Quiminal C. (eds.), Les lois de l'inhospitalité : Les politiques de l'immigration à l'épreuve des sans-papiers, Paris, La Découverte, 1997.

25. Réa A., «Les transformations des régimes de migration de travail en Europe » in Morice A., Potot S. (eds.), De l'ouvrier immigré au travailleur sans papiers. Les étrangers dans la modernisation du salariat, Karthala, 2010, p. 307-315, (p. 310).

26. Castel R., Les métamorphoses de la question sociale. Une chronique du salariat, Paris, Gallimard, 1999 (1995). 
où prédomine le chômage de masse, l'augmentation de l'emploi précaire et la remise en cause de l'État social. L'analyse que fait Robert Castel de cette transformation reste surtout valable pour un pays comme la France mais fonctionne néanmoins pour l'Espagne où, malgré une histoire politique et économique différente, persiste le plus fort taux de travail temporaire en Europe : $32,5 \%$ en 2004 , soit plus d'un tiers des actifs ${ }^{27}$. Ce taux, qui aurait plus que doublé depuis 198528 , atteindrait en 2006 presque $60 \%$ pour les immigrés 29 .

Lorenzo Cachón 30 évoque à ce titre une « discrimination institutionnelle » sur le marché du travail qui serait liée "aux normes publiques en vigueur » et "présente aussi dans les pratiques administratives restrictives quant à l'interprétation ou quant à l'application de ces normes ». De plus, les trois-quarts des immigrés non communautaires étaient concentrés dans quatre branches d'activité : bâtiment, agriculture, hôtellerie et service domestique ${ }^{31}$. Or pour ces migrants de l'époque "postfordiste 32 ", il s'agissait de subir et d'affronter une "discrimination dans l'entreprise 33 » dans un contexte de forte vulnérabilité puisque, en créant une catégorie de travailleur «sans papiers », les politiques d'immigration ont sans doute rompu les derniers remparts qui permettaient aux travailleurs immigrés d'accéder au droit du travail, au syndicalisme et plus généralement à l’État social.

Luis et Manuel n'avaient pas signé de contrat de travail, bien que cela ne soit pas le cas de tous les sans-papiers en Europe ${ }^{34}$. Cette absence de contrat permettait à Raoul de les payer en deçà du salaire pratiqué habituellement sur les chantiers et lui donnait l'opportunité de se débarrasser si besoin des maçons sans devoir en référer aux autorités administratives et juridiques compétentes. En outre, Luis et Manuel n'avaient jamais été maçons avant leur venue en Espagne. Leur incompétence en matière de maçonnerie les rendait difficilement employables dans les autres chantiers et accentuait ainsi leur dépendance à l'égard de Raoul.

Les actions et les discours de Luis et Manuel s'accomplissaient donc dans un contexte politique et socio-économique qui les rendait fortement dépendants de leur chef. Pourtant, au fil des jours, je me rendis compte que leurs

27. Selon l'étude de la Commission européenne « Employment in Europe (1985-2005)».

28. En 1985, il s'élevait à 15,6\%.

29. Selon une étude de l'entreprise de travail temporaire «Adecco ».

30. Cachon L., "L'immigration en Espagne (1996-2006) : logique de marché et “institutionnalisation" ", Travail et Emploi, 115, 2008, pp. 81-93.

31. Ibid.

32. Réa A., Tripier M., "L'immigration ouvrière en France, du "gastarbeiter" au "sans papiers" ", Communication à la Journée d'étude franco-italienne "Immigration et marchés du travail », 8 avril 2008, Aix en Provence.

33. Cachon L., « L'immigration en Espagne (1996-2006) : logique de marché et « institutionnalisation " ", op. cit.

34. Barron P., Bory A., Tourette L., Chauvin S., Jounin N., On bosse ici, on reste ici! La grève des sans-papiers : une aventure inédite, Paris, La Découverte, 2011. 
perceptions de genre, de classe et de race ne découlaient pas mécaniquement de ces structures politiques et socio-économiques et qu'elles n'en étaient pas le strict reflet. Les ethnométhodologues ${ }^{35}$ nous ont déjà appris en effet que l'ordre social n'est jamais posé a priori mais qu'il est négocié, constitué et accompli dans la pratique par les acteurs eux-mêmes. Autrement dit, si les dispositifs juridiques et politiques restreignent les jeux d'acteurs, ils n'en présupposent jamais totalement le déroulement ni l'issue. C'est pourquoi il convient de faire la description des scènes où mes collègues maçons et moi-même jugions et catégorisions l'ensemble de nos discours et de nos pratiques. Ces assignations identitaires auxquelles participaient l'anthropologue et ses hôtes nous renseignent en retour sur l'image qu'ils entendaient donner d'euxmêmes.

\section{La vaillance et la force : marquer les rapports de classe par le genre}

La vie quotidienne sur le chantier avait fourni à mes collègues de travail l'occasion d'un travail d'étiquetage. Je les entendais souvent médire au sujet des maçons espagnols. Ils les considéraient comme fainéants et trouvaient injuste qu'ils puissent bien gagner leur vie tout en travaillant peu d'beures. Au départ, je n'ai pas considéré nécessaire de me sentir concerné par ces remarques. D'abord parce que je n'étais pas espagnol; et puis surtout parce que je travaillais auprès d'eux et que, par conséquent, je m'estimais en quelque sorte inattaquable. Pourtant, je compris progressivement qu'ils n'avaient peut-être pas exprimé ces idées sans penser à moi et qu'il était même tout à fait probable qu'ils m'incluent de fait dans cette catégorie de " maçons espagnols ». Sur le chantier, le moindre repos que je prenais, la moindre grimace que dans l'effort je concédais, la moindre plainte vis-à-vis d'une quelconque douleur, étaient toujours susceptibles d'être interprétés comme le trait d'un caractère faible et paresseux. Luis n'était pas très regardant sur les horaires. Il effectuait souvent des heures supplémentaires dont il était plutôt fier. Tous les matins, lorsque je poussais la porte du chantier, il s'empressait d'étaler sous mes yeux le résultat du travail de la veille qu'il avait effectué en mon absence, c'est-à-dire au moment où j'exerçais, au sein du Forum des cultures ${ }^{36}$, un métier bien moins éreintant que le sien. Manuel participait également à cet étiquetage dévalorisant. Un jour où je lui faisais remarquer les diverses ampoules qui recouvraient mes mains, il me répondit par un sourire narquois. Fallait-il qu'il prenne au sérieux des blessures aussi superficielles? À ses yeux, mon statut d'européen me plaçait d'emblée du côté des "chochottes ", des "engourdis " et des "lèvetard ». "Lave-moi ceci, achète-moi ça, racle le sol, viens m'aider... » aimaient

35. Garfinkel H., Recherches en ethnométhodologie, Paris, PUF, 2007.

36. Ouvert du 9 mai au 26 septembre 2004, le «Forum des Cultures » de Barcelone était articulé autour de trois axes thématiques: «la diversité culturelle et le dialogue des cultures », « le développement durable » et « les conditions pour la paix ». Les contenus du Forum se sont déclinés à travers des conférences, des expositions et des spectacles. Mon travail consistait à accueillir les visiteurs et à les orienter à l'intérieur du site. 
m'ordonner Luis et Manuel. Quant à moi, j'acceptai d'être "l'européen » du chantier, ce que j'étais compte tenu de ma situation administrative, mais je mis un point d'bonneur à ne pas devenir leur homme à tout faire, assigné à des tâches d'emplette et de nettoyage. Pour ce faire, je n'eus d'autres opportunités que de réagir à cette distanciation forcée. Je commençai par refuser de leur acheter le matériel dont ils avaient besoin. Ensuite, j'imposai un peu plus de rythme à mes gestes, dans l'espoir de leur confirmer qu'ils n'étaient pas plus vaillants que moi.

Il y avait dans cette mise à distance à laquelle j'étais confronté tout un jeu autour de la virilité. La façon dont Luis et Manuel me catégorisaient empruntait au registre du genre ${ }^{37}$. Autrement dit, c'est dans le prisme d'une opposition genrée que mes camarades signifiaient et renversaient les rapports de pouvoir dans lesquels, malgré moi, je les avais embarqués. Leur partition entre « eux » et « moi » renvoyait à une opposition entre les travaux nécessitant la force et la technique et ceux relatifs au nettoyage, entre le courage de ceux qui travaillent toute la journée et la faiblesse de celui qui ne peut travailler qu'une demi-journée, entre les corps résistants et solides des « vrais » travailleurs et le corps blessé, défaillant et délicat de celui qui n'est pas fait pour ça. Les qualités féminines, auxquelles j'étais assigné, désignaient et exprimaient mon statut social «d'étudiant européen ». Les rapports de classe étaient ainsi perçus et parlés dans le langage du masculin et du féminin. Stéphanie Gallioz ${ }^{38}$ a déjà montré que la « force physique » est un élément structurant des stéréotypes de genre dans le monde du bâtiment. En outre, elle montre que dans un contexte de féminisation du secteur, les stéréotypes de sexe comme la «force physique masculine », l'«incompétence technique des femmes », la « courtoisie masculine » et la «minutie féminine » ${ }^{39}$ peuvent paradoxalement tourner à l'avantage des femmes dans la division sexuelle du travail puisque celles-ci se voient confier les travaux « les moins pénibles et (les) plus visibles aux yeux des clients ${ }^{40}$. En revanche, sur les chantiers de Raoul, dans un contexte de travail homosexué, les stéréotypes de sexe ne favorisaient pas les hommes comme moi renvoyés par les autres à des marqueurs construits comme «féminins ». Pour réaffirmer une forme de masculinité, je n'avais d'autres choix que de prouver mon aptitude physique en exerçant de pénibles travaux de force. Très vite, je me surpris à faire preuve de quelques excès de zèle sur les chantiers, comme lorsque, par exemple, je mis un point d'honneur à briser seul et en une seule journée, au marteau et à la pelle, le carrelage et la dalle d'un appartement. Cet exercice constituait une véritable " performance de genre " ${ }^{41}$ si l'on en croit la réaction de Raoul qui, d'abord étonné par ma

37. Scott W. J., "Genre : une catégorie utile d'analyse historique ", Les Cabiers du GRIF, 37-38, 1988; pp. 125-153.

38. Gallioz S., "Force physique et féminisation des métiers du bâtiment », Travail, genre et sociétés, 16-2, 2006, pp. 97-114 et Gallioz S., « La féminisation des entreprises du bâtiment : le jeu paradoxal des stéréotypes de sexe ", Sociologies pratiques, 14-1, 2007, pp. 31-44.

39. Ibid, p. 43.

40. Ibid, p. 43. 
prouesse, avait tenu à me féliciter. Je venais de démontrer à Raoul, Luis et Manuel une certaine capacité au travail physique. Mais était-ce suffisant pour qu'ils me considèrent comme l'un des leurs ?

\section{Authenticité et économie contre facticité et gaspillage. Comprendre l'anti-européanisme ordinaire}

Au début du mois de mai, tandis que nous venions de mettre de l'ordre en jetant un tas de débris, nous décidâmes d'un commun accord de prendre une courte pause. Nous étions tous assis au milieu du chantier, en train de nous requinquer avec des tranches de salami et quelques gorgées de jus d'orange. Je regrettai de jeter le beau miroir dont nous venions de dévisser les dernières attaches en précisant que nous aurions pu le revendre aux puces. Luis : "ouais c'est $d u$ gâchis... mais ici, en Europe, ils balancent tout, ils polluent bien plus que chez nous". Manuel acquiesce d'un signe de tête aux dires de Luis. Luis : "quand ils voient le moindre défaut sur un objet, ils le foutent à la poubelle...". Manuel (en se tournant vers moi) : "tu sais, là-bas c'est différent, nous, on rafistole tout ce qu'on trouve, on ne jette rien". Yann: " mais vous savez, en Europe aussi ça leur arrive de réparer des objets... ". Luis : "non mais c'est quand même pas pareil, chez nous, l'esprit est différent tu comprends, comme les gens n'ont pas d'argent, ils n'ont pas le choix... ". Tout à coup, je me sentis mis à l'écart. Non seulement je n'étais pas dans le "nous " que venait d'utiliser Manuel mais de surcroît les deux hommes m'avaient vêtu d'un habit culturel taillé à ma mesure : j'étais l'européen "gaspilleur " et "pollueur". Subrepticement, mes hôtes venaient de rappeler ma différence.

L’idée d'une Europe située loin des préoccupations environnementales et allant à contre-courant $\mathrm{du}$ « naturel » revenait souvent dans nos discussions, notamment à propos de la nourriture. Ce qui me heurtait et m'affligeait grandement, c'était la certitude avec laquelle ils m’incluaient dans ce monde européen fait d'aliments synthétiques et factices. Je représentais pour eux l'homme occidental entretenant un rapport inauthentique à la vie.

Manuel s'était ainsi imaginé que je n'avais jamais gô̂té aux véritables jus de fruits, c'est-à-dire directement pressés, ne les connaissant que sous forme de bouteilles industrielles. Un jour, il en fit part à un nouvel ouvrier sur un ton moqueur. Luis pensait quant à lui que je ne mangeais que des boîtes de conserves et que l'art culinaire m'était inconnu.

L'image d'européen qu'il me tendait ne me convenait pas, je n'étais pas riche et surtout je ne me considérais pas comme un consommateur passif ou gaspilleur. Je leur rappelai que mes conditions de vie n'étaient pas beaucoup

41. Butler J., Trouble dans le genre, Le féminisme et la subversion de l'identité, Paris, La Découverte, 2005. 
plus envieuses que les leurs. J'avais rencontré Manuel en sous-louant une chambre dans un appartement délabré. En raison de notre faible budget, ma compagne et moi avions dû nous tourner vers le marché de la sous-location et, au sein de celui-ci, vers les offres les moins chères ${ }^{42}$. Ainsi, ma rencontre avec Manuel n'avait pas été programmée en raison d'un projet ethnographique sur les maçons sans-papiers. En revanche, nous avions sous-loué des chambres de même nature car nos conditions matérielles d'existence étaient similaires. $\mathrm{Ni}$ ma façon de m'habiller, ni mes habitudes alimentaires ne témoignaient d'une quelconque richesse. Je décidai d'informer encore Luis et Manuel de mes difficultés à financer ma thèse. À ce titre, tous les deux savaient que mon activité sur les chantiers servait aussi à me nourrir et me loger. En leur rappelant mes conditions de vies pour le moins précaires et voisines des leurs, je cherchais à atténuer les symboles raciaux et sociaux attachés à ma nationalité.

Il résidait cependant une limite essentielle dans mon entreprise de légitimation sociale. Car si je n'étais pas « riche », je demeurais un « européen » autorisé à vivre en Espagne. Je n'étais donc pas exactement le «bourgeois inauthentique » à qui ils pensaient mais sans être pour autant le « pauvre sanspapiers » qu'ils étaient, d'où le «trouble 43 » que constituait ma présence. J'étais un ouvrier précaire dont l'argent du chantier servait aussi à vivre mais j'étais aussi un ethnographe français. Il y avait sans nul doute pour mes hôtes matière à discussion. Je perturbais leur « partage du sensible », pour reprendre l'expression de Jacques Rancière ${ }^{44}$, en ce que je n'étais pas à la place qui me revenait d'ordinaire en tant que «blanc », «français » et « intellectuel ». C'est parce que Luis et Manuel me percevaient en tant qu' « européen » et « intellectuel », mais aussi en tant que " précaire » voire «pauvre », que ma présence sur les chantiers constituait pour eux une forme de «breaching experience 45 »: en introduisant de la perturbation et de l'étrangeté, ma participation à l'entreprise de Raoul permettait de révéler des « allant de soi » liés à l'organisation du travail des sans-papiers. Elle suscita ainsi des comportements adaptatifs de la part de chacun d'entre nous, qui mirent en relief par défaut les normes sociales en vigueur sur le chantier en matière de genre, de classe et de race. Mon intégration au sein de leur univers professionnel impliquait aussi ce que Everett Hugues nomme des « dilemmes et contradictions de statut 46 ». Hugues propose en effet d'examiner « les conséquences de l'arrivée de nouvelles catégories de personnes dans des positions déjà définies ». Il ajoute que " tout événement de ce type produit dans une certaine mesure une contradiction de statut, et peut également créer un dilemme de statut pour l'individu concerné et pour ceux qui ont affaire à lui ». Hugues prend l'exemple d'un « noir » « qualifié

42. Le loyer d'une chambre double (pour un couple) dans un appartement confortable s'élevait au moins à 450 euros/mois (sans compter les charges).

43. Butler J., Trouble dans le genre, op. cit.

44. Rancière J., Le partage du sensible. Esthétique et politique, Paris, La Fabrique, 2000.

45. Garfinkel H., Recherches en ethnométhodologie, Paris, PUF, 2007.

46. Hugues E., « Dilemmes et contradictions de statut », in Hugues E., (ed.), Le regard sociologique, Paris, Éditions de l'EHESS, 1996. 
pour une des professions établies ", à savoir le cas d'un médecin noir. Pour lui, "le dilemme, pour les Blancs qui le rencontrent, réside dans le choix entre le traiter comme Noir ou comme membre de sa profession ». Qu'en est-il de l'anthropologue blanc qui devient ouvrier dans un monde professionnel où ne travaillent que des «non-blancs » ? Dans mon cas, il y avait même un double dilemme de statut, l'un concernant l'appartenance au monde des «blancs », l'autre lié au statut d'intellectuel. Comment mes collègues de travail « nonblancs » devaient-ils considérer un ouvrier «blanc »? Et comment des ouvriers devaient-ils considérer un ouvrier par ailleurs anthropologue ?

Si la double qualification de "blanc » et d' "intellectuel » avait parfois pour moi quelques avantages, mes compagnons de labeur ne pouvaient quant à eux en bénéficier. Dès lors, ils n'avaient jamais tout à fait accepté ma plus grande liberté devant le travail et les quelques traitements de faveur que m'accordait Raoul. Aussi, quelle avait été leur surprise lorsque je leur avais annoncé que le chef était disposé à m'embaucher à mi-temps. D'autant que ce dernier allait me payer cent euros la moitié de semaine tandis qu'eux ne recevaient que 180 euros pour une semaine complète. "Mais pourquoi travailles-tu à mi-temps, je croyais que tu avais besoin d'argent? ", avait fait judicieusement remarquer

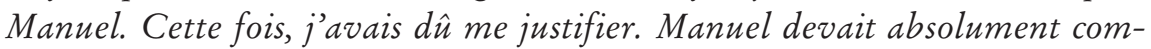
prendre que je ne travaillais pas sur les chantiers pour épargner de l'argent, c'est-à-dire pour les mêmes raisons que lui. J'espérais simplement qu'il prenne pleinement conscience de ce qui nous séparait. Pourtant les refus répétés que je formulais à l'encontre de l'image que me tendaient mes collègues de travail étaient tous voués à l'échec, ne serait-ce qu'en raison du respect gêné que témoignait Raoul à mon égard, qui ne passait jamais inaperçu et qui offrait à Luis et Manuel la preuve irréfutable que je n'étais pas "eux".

Nos rapports sociaux s'étaient donc progressivement stabilisés autour de ce trouble qu'avait jeté initialement dans le chantier mon statut de « maçon français ethnologue ». Ma bonne volonté n’y suffisait pas car je n'étais pas le même maçon que Luis et Manuel. Je ne tenais pas les truelles pour les mêmes raisons. Je les tenais dans le cadre d'une enquête ethnographique, tandis qu'ils les tenaient pour épargner l'argent nécessaire à leur projet migratoire. L'incongruité de ma présence rendait davantage visibles l'ensemble des discriminations dont ils souffraient. En étant là, j’offrais la possibilité d'une comparaison qui objectivait leurs positions.

Luis et Manuel étaient contraints de travailler au sein de chantiers sales et dangereux, dans l'attente d'une partie de leurs salaires. Plus les jours passaient, plus nos conditions de vie tendaient encore à se distinguer. L'administration $d u$ Forum des Cultures venait de me recruter pour cinq mois et désormais le chantier ne constituait plus mon unique source de revenus. Ce nouvel emploi me permit de réduire encore mes heures passées sur les chantiers. Bientôt, je ne travaillais plus que trois matinées par semaine à leurs côtés. Comment, devant de 
telles différences, pouvais-je encore être étonné des réactions de Luis et Manuel. Je les vexais, je les agaçais, je les provoquais, sans en avoir l'ambition, lorsque je leur narrais les soirées que je passais an Forum des cultures, quand je leur décrivais mon appartement situé an coeur du quartier de Gracia, on bien lorsque j'évoquais ma rencontre à Paris avec mon directeur de thèse et mes prochaines vacances en France chez mes parents. Pendant ce temps, ils se demandaient à quel moment et de quelle façon ils pourraient obtenir des papiers, trouver un emploi plus digne, rendre visite à leurs familles ou reprendre des études.

Leurs remarques sarcastiques renvoyaient à ma présence sur de tels chantiers, dont nous savions tous en réalité qu'elle dépendait de mon projet ethnographique. Si je m'étais astreint à mon simple rôle d'étudiant Erasmus, je me serais certainement efforcé d'exercer un autre métier que celui de maçon. Luis et Manuel en étaient bien sûr persuadés et c'est à ce titre que je représentais à leurs yeux une sorte d'imposture. Leurs railleries consistaient à rompre le mensonge. À travers elles s'exprimait l'idée d'une importante différence entre eux et moi. Car pour Luis et Manuel, je demeurais l'étudiant français disposant de papiers et libre de quitter les chantiers de Raoul. Ainsi, nos différences ne reposaient pas sur les prétendus traits culturels qu'ils évoquaient sans cesse. Je souhaitais surtout que Luis et Manuel puissent se convaincre que leur « nous » existait, mais sûrement d'une autre façon qu'ils se l'étaient imaginés. Au fil de l'enquête, je compris aussi que le «nous » qu'ils utilisaient devant moi était à la mesure de l'accueil stigmatisant qui leur était réservé et, en particulier, de leur expérience des discriminations raciales.

\section{Les faveurs de « race ». Des nationalités racialisées.}

Mes collègues ouvriers et moi, nous n'étions pas logés à la même enseigne. À la différence de Luis et Manuel, je pus d'emblée adapter mes horaires de travail et je ne dus jamais effectuer d'beures supplémentaires non rémunérées. Par ailleurs, Raoul était dur avec eux. Il leur parlait sans ménagement, ou plutôt, il ne leur parlait pas, il leur donnait des ordres, tandis que je me demandais souvent pourquoi Raoul n'osait jamais me regarder dans les yeux. En dépit de la médiocrité de mes travaux, je n'eus jamais à subir de fortes remontrances de la part du chef, à la différence de mes collègues de travail qui en firent les frais quotidiennement. Son timbre de voix n'avait rien d'agressif quand il m'adressait la parole. "C'est parce que toi, il te respecte, tu es Français ... » avait habilement relevé Luis. Il existait donc une catégorisation des travailleurs fondée sur une biérarchie racialisée des nationalités 47 qui impliquait un ensemble d'attitudes différenciées de la part du chef. Mais l'attention estimable que me témoignait Raoul ne constituait pas la seule expression de nos différences. $\mathrm{Ma}$

47. Fassin D., Fassin E. (eds.), De la question sociale à la question raciale ? Représenter la société française, Paris, La Découverte, 2006. 
nationalité française m'avait d'emblée autorisé à bénéficier d'un salaire proportionnellement plus élevé que Luis et Manuel, sans même que je n'eus d'ailleurs à le négocier. Raoul me versait en quelque sorte le salaire du "Français" ou ce que l'on pourrait appeler, à la suite de Roediger, le "salaire de la blanchité $48 »$.

L'expérience de la discrimination raciale, à laquelle je ne fus jamais confronté, unissait mes collègues de travail, au-delà de la diversité de leurs nationalités. Leur «nous» signifiait davantage cet écart de traitement entre « eux » et « moi » qu'une supposée culture «sud-américaine » qui me serait essentiellement extérieure. Luis et Manuel n’avaient pas eu à négocier pour être embauchés et vivre de telles conditions de travail et la présence d'un Français sur ce type de chantiers restait pour eux un événement fort inhabituel.

Avant même que Raoul ait accepté mes services, Luis et Manuel avaient d'ailleurs exprimé quelques réserves à ma volonté d'intégrer les chantiers : "ce n'est pas fait pour toi ", avaient-ils averti à plusieurs reprises. Maria, l'une des résidentes qui vivait au dessus du chantier était du même avis. Le 7 mai 2004, la chaleur et les mauvaises odeurs me poussaient à ouvrir en grand la porte $d u$ logement. Quelques minutes plus tard, je la vis arriver avec son fils Jorge; tous les deux scrutaient minutieusement l'état des travaux. Les ayant immédiatement salués, je pensais que la mère et le fils passeraient leur chemin mais leurs regards se firent au contraire plus insistants.

Yann: "vous avez besoin de quelque chose?".

Jorge : "non, non, on voulait juste voir comment c'était».

Maria : "qu'est-ce que vous faites exactement?".

Yann: "on va faire un logement".

Jorge : "avec le bruit que vous faites, on se demandait ce qui se passait".

Yann: "je suis en train de retirer du platre vous voyez".

Jorge : "c'est-à-dire qu'on a quand même peur pour le plafond ».

Yann: "le plafond?".

Jorge : "oui, parce que le plafond c'est notre plancher, et comme vous tapez fort...».

Maria : "on habite au-dessus vous savez, il faut faire attention, ça peut s'écrouler».

Yann : "oui, oui, je comprends, ça fait beaucoup de bruit mais rassurez-vous, Raoul a l'babitude, il sait ce qu'il fait».

Maria: "vous êtes Péruvien vous aussi?"

48. Roediger D., The Wages of Whiteness: Race and the Making of the American Working Class, Londres/New York, Verso, 1999. David Roediger emprunte à William E.B. Du Bois, un sociologue « noir » de la première moitié du vingtième siècle, son titre : "the wages of whiteness »; c'est « le salaire de la blanchité », soit le privilège de la couleur qui reste aux classes populaires «blanches » quand leur statut social est dévalué : « je n’ai pas grand-chose, mais au moins je suis blanc». 
Yann: "non, non, je suis Français».

Maria : "ah, j'aime mieux ça».

Yann: "abbon».

Maria : "parce que les autres ils sont Péruviens non?"

Yann: "certains».

Maria : "non mais Raoul, il est Péruvien?"

Yann: "oui... enfin maintenant il est Espagnol».

Maria : "oui mais où il est né ?... Au Pérou non?"

Yann: "oui, oui...»

Maria: "...mais alors qu'est-ce que vous faites là si vous êtes Français, vous pourriez trouver mieux que ça quand même. Pourquoi vous ne travaillez pas en France, la France, c'est quand même mieux que l'Espagne, il y a plus de travail là-bas... ».

Au cours de cette brève interaction, Maria ne comprend pas 1) pourquoi un « Français » est venu travailler en Espagne et 2) pourquoi un «Français » travaille dans une entreprise du bâtiment dont le chef est un « Péruvien ». La première interrogation de Maria renvoie aux différentes vagues d'immigration espagnoles qui se sont succédées en France tout au long du Xxème siècle. L'histoire récente nous a en effet davantage habitués à rencontrer des maçons espagnols en France plutôt que des maçons français en Espagne. Quant à sa seconde remarque, elle fait référence à une discrimination raciale qui organiserait le monde professionnel du bâtiment. Maria n'est pas habituée à rencontrer des maçons «français » en Espagne et surtout pas sur de tels " petits » chantiers, c'est-à-dire en un lieu qui est en quelque sorte réservé aux immigrés et plus particulièrement aux immigrés sans papiers. Les illégaux occuperaient selon elle les petits chantiers masqués aux passants, comme celui qui est en bas de chez elle. Les chantiers de Raoul étaient en effet différents des grands chantiers magnifiés des grands groupes du BTP, comme ceux qui pouvaient être contemplés en bordure des grandes avenues et d'où dépassaient d'immenses grues. Dans les quartiers populaires, comme celui où vit Maria, les travaux de réhabilitation comme les ouvriers étaient rendus invisibles tandis que dans les grandes artères touristiques, les grands travaux participaient de la mise en scène d'une ville dynamique; ils étaient à ce titre exposés à la vue de tous à travers des panneaux sur lesquels de grandes fresques décrivaient le projet architectural. Le point de vue de Maria ne signifie pourtant pas qu'il n'y a jamais eu de sans papiers œuvrant sur les chantiers officiels mais il indique en revanche qu'il est fort inhabituel de rencontrer des «Espagnols » ou des « Français » sur des chantiers comme ceux de Raoul. «Français » et « maçon », je représentais pour Maria une forme d' « anomalie taxinomique 49 ». Non pas en raison de deux catégories cognitives essentiellement incompatibles, mais parce que le contexte historique lié à l'immigration illégale en Espagne rendait ma présence

49. Douglas M., De la souillure, Paris, Maspero, 1971 (Réédition en 2001 aux éditions La Découverte). 
au sein de ce décor professionnel étonnante, voire troublante. Aux yeux de Maria, ce chantier n'était pas digne de mon rang, de ma nationalité. Par ailleurs, elle était perturbée qu'un Péruvien puisse diriger un Français (bien qu'elle méconnaissait l'ensemble des avantages dont je bénéficiais). À la fin de la conversation, Maria mobilise ainsi une catégorie racialisée (« Péruvien ») en vue de délégitimer une position sociale par ailleurs perçue comme respectable (« chef d'entreprise »).

\section{La référence raciale sur des petits chantiers informels du bâtiment}

De nombreux sociologues ont déjà analysé le racisme au travail 50. La plupart s'accordent pour dire que les attitudes et les pratiques racistes ne sont pas importées depuis l'extérieur dans l'entreprise mais qu'elles sont consubstantielles à son organisation interne. Nicolas Jounin écrit à ce titre : « le racisme n’apparaît pas comme dérive ou débordement de préjugés retardataires fabriqués en dehors de l'entreprise. Il intervient comme partie intégrante de l'activité de l'entreprise » 51 . En lisant ces auteurs, il apparaît que les logiques de classification raciales organisent la gestion de la main-d'œuvre, son recrutement, sa répartition, sa rémunération et son avancement. Déjà dans L'établi ${ }^{52}$, Robert Linhart, au terme d'une observation militante chez Citroën, en donnait une description éclairante : « quant à la répartition, elle se fait d'une façon tout à fait simple : elle est raciste. Les noirs sont M1, tout en bas de l'échelle. Les Arabes sont M2 ou M3. Les Espagnols, les portugais et les autres immigrés européens sont en général OS1. Les Français sont d'office OS2, et on devient OS3 à la tête du client, selon le bon vouloir des chefs » ${ }^{53}$. Plus récemment, Nicolas Jounin relève dans le secteur du BTP d'Île-de-France une ethnicisation des rapports de travail qui aboutit à l'assignation des africains à des tâches de manœuvres alors que les Portugais deviennent «chefs ». Cette " gestion ethnique » des travailleurs contribue selon lui « à réduire l'incertitude et à ordonner les statuts sur le chantier » ${ }^{44}$ dans un contexte où, précisément, il existe sur un même chantier une multiplicité d'employeurs et donc une grande variété de statuts pour les ouvriers. Les chantiers de Raoul présentaient à ce titre une toute autre configuration.

50. Bataille P., Le Racisme an travail, La Découverte, Paris, 1997 ; De Rudder V., et Vourc'h F., «Les discriminations racistes dans le monde du travail », in Fassin E., Fassin D. (eds.), De la question sociale à la question raciale, op. cit., pp. 175-194 ; Linhardt R., L'Établi, Paris, Minuit, 1978 ; Jounin N., Chantier interdit au public. Enquête parmi les travailleurs du bâtiment, Paris, La Découverte, 2008.

51. Jounin N., «L'ethnicisation en chantiers. Reconstructions des statuts par l'ethnique en milieu de travail ", Revue européenne des migrations internationales, 20-3, 2004, pp. 103-126.

52. Linhardt R., L'Établi, op. cit.

53. Ibid, p. 24.

54. Jounin N., «L'ethnicisation en chantiers. Reconstructions des statuts par l'ethnique en milieu de travail », op. cit. 
Au moment où je travaillais sur les chantiers, lorsque Raoul s'adressait à ses ouvriers pour juger leurs travaux, il ne faisait pas non plus référence à leurs caractéristiques raciales. En revanche, lorsque je lui rendis visite sur l'un des chantiers, six mois après mon départ, Raoul me fit la description d'une " gestion ethnique des travailleurs $» 55$.

En arrivant sur le chantier, je ne reconnaissais pas un seul de ses ouvriers. La plupart avaient quitté son entreprise suite à de nombreux impayés et Raoul avait procédé à un total renouvellement de la main d'œuvre. Lorsque je lui demandai pourquoi il n'avait plus les mêmes, il répondit qu'il n'avait plus "la même philosophie " (Raoul). Selon lui, s'il n'avait pas payé en temps voulu ses anciens ouvriers, c'est parce qu'ils n'étaient pas assez compétents et qu'ils n'avançaient pas assez vite les travaux. Son argumentation reposait sur les principes suivants : les logements n'étant pas livrés à la date prévue, les propriétaires tardaient à le rémunérer; dès lors, il ne disposait plus du budget nécessaire pour rémunérer ses ouvriers.

Raoul: "c'est pour ça que maintenant je prends seulement des gens compétents, des professionnels, sinon c'est trop de problèmes».

Yann: " mais ça doit te revenir plus cher?"

Raoul: "oui, ils me coûtent plus cher, mais comme ils travaillent vite et bien, j'en ai moins, donc c'est plus rentable. Lui, tu vois, il est maestro, il sait tout faire... c'est un chinois, c'est un bosseur, je suis obligé de lui dire d'arrêter, sinon il ne prendrait même pas sa pause pour manger, il travaille jusqu'à ce qu'il soit complètement épuisé».

Ainsi, si dans cette petite entreprise illégale du bâtiment, il y a bien une gestion ethnique des travailleurs, en revanche, elle ne prend pas racine dans un contexte d'incertitude concernant les statuts mais dans un contexte d'incertitude concernant les compétences des maçons. En recrutant un « chinois» «bosseur » qui «sait tout faire », Raoul prétend avancer plus rapidement les travaux, respecter les délais fixés par les propriétaires et payer plus régulièrement ses ouvriers. Les propriétés raciales des ouvriers fondent ici le choix d'une nouvelle stratégie entrepreneuriale de la part de Raoul.

\section{Les maçons «sud-américains » versus les maçons « espagnols »}

Au moment de l'enquête, la plupart des maçons qui travaillaient pour Raoul étaient Sud-américains (Paraguayen, Péruviens, Équatoriens). Un seul était Roumain. Ces maçons entraient souvent dans des jeux de moquerie où ce qui était raillé était l'origine ou l'apparence « indienne » de tel ou tel ouvrier. Ayant tous potentiellement un quelconque lien avec une supposée culture " indienne ", chacun pouvait tour à tour être moqueur et moqué. L'ouvrier moqué l'était généralement en raison d'un attribut «indien » (une apparence, 
une origine, une langue) qui dénotait un esprit archaïque et dépassé en totale opposition avec l'esprit moderne réputé quant à lui éclairé et favorable au progrès. Mais si mes collègues de travail mettaient en avant une origine ou une apparence indienne dans le cadre d'insultes " amicales », en revanche ils ne l'appliquaient jamais pour évaluer leurs pratiques professionnelles. Les ouvriers de Raoul ne parlaient donc pas de «travail d'Indien » comme les maçons en France parlent de « travail d'Arabe » 56.

En revanche, comme on l'a vu plus haut, les ouvriers de Raoul se présentaient volontiers comme des " maçons sud-américains » pour mettre en avant des qualités professionnelles (endurance, ardeur au travail) dont les « maçons espagnols » et européens seraient d'après eux dépourvus. Ainsi, dans un contexte de relative homogénéité statutaire (la plupart étaient manœuvres et tous travaillaient sans contrat de travail), les maçons (tous sud-américains) ne mobilisaient pas la référence ethnique dans le cadre d'une différenciation professionnelle interne au chantier mais plutôt pour se différencier des maçons « espagnols » travaillant sous-contrat dans des conditions de travail et de rémunération plus avantageuses. Sans-papiers et peu qualifiés, les grands chantiers du BTP espagnols étaient inaccessibles à mes collègues de travail. Ainsi, c'est dans le contexte d'un marché du travail fortement segmenté qu'ils considéraient les maçons espagnols comme des «fainéants » tous plus ou moins prébendiers.

\section{La référence raciale : le «texte caché » des maçons sans-papiers}

Sur le chantier, Raoul n'était pas exempté des considérations raciales, même si ces dernières ne lui étaient jamais directement adressées. De petite taille, 1,60 m environ, la peau mate, les cheveux et les yeux noirs, Raoul était identifié comme un indien par mes collègues de travail. Souvent je le vis gérer les attributs " discrédités 57 » qui rappelaient sa différence et qui pouvaient à ce titre mettre en cause son autorité. Lorsqu'il n'était pas là, Luis et Manuel aimaient dénigrer ses traits phénotypiques.

Ces considérations raciales advenaient toujours dans le dos du pouvoir, en tant que « texte caché 58 » qui permettait de contredire les discours et les attitudes de soumission que Luis et Manuel mettaient en scène lorsqu'ils se trouvaient face à Raoul. Ma participation au chantier m'a permis d'accéder à ces actes de résistances non organisés et non structurés et qui échappent souvent aux sociologues dont la méthodologie principale repose sur l'entretien ou l'outil statistique. La description péjorative que Luis et Manuel faisaient de Raoul renvoyait ce dernier à une identité indienne essentielle, prétendument

56. Ibid.

57. Goffman E., Stigmate, les usages sociaux des handicaps, Paris, Éd. de Minuit, 1975.

58. Scott J., La domination et les arts de la résistance. Fragments d'un discours subalterne, Paris, Amsterdam, 2008. 
rurale et en dehors du monde moderne. Cette façon de présenter Raoul était bien évidemment incompatible avec l'image du chef d'entreprise riche et dynamique que Raoul entendait mettre en scène.

Au cours du mois d'avril, Raoul considéra que Luis n'avait pas peint convenablement un mur. Luis, très en colère, attendit que Raoul quitte le chantier pour expliquer aux autres ouvriers: "il fait le chef, il croit être un homme important, mais en vérité, t'as vu sa tronche, c'est qu'un Indien, un vraipaysan ".

Ainsi, Luis racialisait Raoul pour rompre l'assise de son pouvoir. À ses yeux, les pratiques de Raoul n'étaient pas seulement abusives en elles-mêmes mais aussi parce qu'elles étaient le fait d'un «Indien », c'est-à-dire exercées par une personne qui n'en avait pas vraiment la légitimité. À ce titre, l'attitude bienveillante que Raoul adoptait à mon égard ne représentait pas simplement le respect du «blanc » de la part d'un «Indien ». Les précautions avec lesquelles Raoul envisageait notre relation renvoyaient au désir de se considérer lui-même «blanc », c'est-à-dire comme moi : l'autre «blanc » du chantier 59. Dès lors, la mise en scène d'une relation quelque peu égalitaire auprès d'un Français (par ailleurs ethnologue) permettait à Raoul de passer au second plan, voire sous silence, les effets de stigmatisation liés à une apparence physique racialisée.

\section{Gérer le stigmate racial en mettant en scène une appartenance de classe}

Au cours de nos rares interactions, la gestion du stigmate racial consistait pour Raoul à mettre en avant un statut social valorisant. Or cette stratégie n'allait pas de soi, tout particulièrement lorsque je réalisai un entretien enregistré dont il savait qu'il serait utilisé dans le cadre de mon travail universitaire. Car si j'étais le "Français blanc ", j'étais en sus "l'enquêteur-sociologue » qui s'intéressait à la vie des immigrés «sud-américains » en Espagne. Les questions que je lui posais étaient embarrassantes dans la mesure où mon statut d'enquêteur le plaçait dans une entreprise de légitimation sociale. Il était désormais espagnol (et européen) et il souhaitait à ce titre que je le considère comme un entrepreneur du bâtiment "barcelonais ", si possible aussi futé, riche que dynamique. En lui demandant de participer à l'enquête, je proposais une autre définition de la situation. En me présentant non plus comme un ouvrier mais comme un anthropologue, je le plaçais dans une position délicate car je rompais tout à coup la relation d'autorité qui nous liait sur le chantier. À présent, Raoul n'était plus seulement le chef, il était aussi celui qui répondait à mes questions dans le cadre d'une enquête ethnographique sur des immigrés dont il souhaitait absolument se distinguer. En l'incitant à justifier sa différence par rapport aux ouvriers immigrés, je participais, sans en avoir 
pourtant l'ambition, au nivellement de notre relation. «L'autorité ethnographique 60 », projetée dans un texte à venir, tempérait ici, voire renversait, celle dont Raoul pouvait bénéficier au sein du chantier. Lors des entretiens, Raoul utilisait un langage plus soutenu, des mots dont il pensait qu'ils seraient mieux compris par ce «Français sociologue » à qui il devait prouver et attester qui il était. Ainsi, Raoul, le « Péruvien espagnol », justifiait son excellence et sa probité face à l' "étudiant français », même si l'image édulcorée qu'il me tendait était quelque peu ternie par les retards de salaires auxquels il habituait ses ouvriers et par la situation économique pour le moins défavorable de son entreprise.

\section{Jeux de miroir et de pouvoir dans une petite entreprise illégale du bâti- ment}

La situation professionnelle que j'observai impliquait donc une complexité des catégorisations de genre, de classe et de race. Soit l'on parlait de race à travers la culture, comme le firent Luis et Manuel afin de mettre à distance un anthropologue Français inopinément ouvrier ou un chef perçu comme illégitime, soit au contraire l'on cherchait comme Raoul à s'extraire de la race pour conforter une position sociale chèrement acquise et quelque peu mise à mal par la situation ethnographique. L'articulation de ces catégories renvoyait donc à une configuration du pouvoir spécifique qui ne pouvait être résumée dans une logique verticale du pouvoir : aussi bien celle d'un chef qui exploite et domine sans merci des ouvriers sans-papiers que celle d'un ethnologue qui impose sans obstacle son enquête à des enquêtés vulnérables. Le chef était un ancien immigré tandis que j'étais un ouvrier «blanc » et « anthropologue ». Dès lors, deux logiques d'identification étaient à l'œuvre sur les chantiers : une logique de rapprochement identitaire et une logique de distanciation identitaire. Raoul souhaitait apparaître foncièrement différent des sans-papiers mais ces derniers contestaient son autorité en le rapprochant d'eux-mêmes, c'est-à-dire en l'amalgamant dans une identité « indienne » à laquelle ils pensaient tous à quelques degrés appartenir. De mon côté, soucieux de réaliser une ethnographie impliquée, j’entendais me fondre dans leur univers professionnel ; or c'est en revendiquant une forme de masculinité et une appartenance culturelle racialisée que Luis et Manuel entendaient invoquer un fossé insurmontable entre nous.

Dans un contexte d'économie informelle où l'absence de contrat de travail et la faible qualification des travailleurs ne réglaient pas explicitement la question de la division des tâches et des relations de subordination, l'ensemble des membres de l'entreprise fabriquaient des relations de pouvoir à partir d'oppo-

60. Clifford J., «De l'autorité en ethnographie. Le récit anthropologique comme texte littéraire » (1983), in Cefaï D. (ed.), L'enquête de terrain, Paris, La Découverte/MAUSS, 2003. L'autorité renvoie ici au texte que j'avais projeté d'écrire. Raoul était bien sûr au courant que mon enquête déboucherait sur une publication. 
sitions de classe, de genre ou de race. Ces rapports sociaux n'étant cependant jamais totalement indépendants les uns des autres, c'est en observant le jeu de leur interpénétration que l'on peut comprendre comment ces rapports dissymétriques tendent à se nuancer, se tempérer voire s'inverser. 